\title{
Fluorescence spectroscopic detection of mitochondrial flavoprotein redox oscillations and transient reduction of the NADPH oxidase-associated flavoprotein in leukocytes
}

Received: 27 May 2003 / Accepted: 27 September 2003/Published online: 23 October 2003

(c) EBSA 2003

\begin{abstract}
Steady-state and time-resolved fluorescence spectroscopy and fluorescence microscopy of leukocyte flavoproteins have been performed. Both living human peripheral blood monocytes and neutrophils have been utilized as experimental models, as the former relies much more heavily on mitochondrial metabolism for energy production than the latter. We confirm previous studies indicating that cellular flavoproteins absorb at $460 \mathrm{~nm}$ and emit at $530 \mathrm{~nm}$, very similar to that of the FAD moiety. Furthermore, the emission properties of intracellular flavoproteins were altered by the metabolic inhibitors rotenone, antimycin A, azide, cyanide, DNP (2,4-dinitrophenol), and FCCP [carbonyl cyanide $p$-(trifluoromethoxy)phenylhydrazone]. Kinetic studies revealed flavoprotein emission oscillations in both monocytes and neutrophils. The flavoprotein intensity oscillations correlated with the physiological status of the cell and the nature of membrane receptor ligation. Microscopy revealed the presence of flavoprotein fluorescence in association with the plasma membrane, intracellular granules and distributed throughout the cytoplasm, presumably within mitochondria. Metabolic inhibitors such as cyanide suggest that the plasma membrane and granular components are cyanide-insensitive and therefore are likely associated with the flavoprotein component of the NADPH oxidase, which is located in these two compartments. This interpretation was found to be consistent with structural localization of the NADPH oxidase using an antibody molecule specific for this protein. Using peripheral blood neutrophils, which display less active mitochondria, and time-resolved emission spectroscopy, we show that the NADPH
\end{abstract}

A. Kindzelskii $\cdot$ H. R. Petty $(\bowtie)$

Departments of Ophthalmology and Visual Science and Microbiology and Immunology, The University of Michigan School of Medicine, 1000 Wall Street, Ann Arbor, MI 48105, USA E-mail: hpetty@umich.edu

Tel.: + 1-734-6470384

Fax: + 1-734-9363815 oxidase-associated flavoprotein undergoes a periodic transient reduction of about $54 \pm 2 \mathrm{~ms}$ in living cells. This finding is consistent with prior studies indicating that propagating substrate (NADPH) waves periodically promote electron transport across the NADPH oxidase.

Keywords Flavoprotein - Leukocyte Mitochondria · NADPH oxidase $\cdot$ Fluorescence spectroscopy

\section{Introduction}

Electron transport systems play vital roles in energy production and in the production of superoxide anions, an important reactive oxygen metabolite (ROM). Flavoproteins participate in (1) mitochondrial electron transport reactions of the NADH dehydrogenase (complex I of the respiratory chain) and (2) superoxide anion formation by the NADPH oxidase, a protein found in granule and plasma membrane fractions of leukocytes. Flavoproteins are also noteworthy because they demonstrate autofluorescence in the oxidized, but not reduced, state (Kunz 1986; Kunz and Kunz 1985; Kunz et al. 1993, 1997, 2002; Kuznetsov et al. 1998). Thus, flavoprotein autofluorescence reflects electron trafficking through the proteins' active sites. Flavoprotein fluorescence has been used extensively to monitor the metabolic status of cells. About one-half of the flavoprotein fluorescence is due to $\alpha$-lipoamide dehydrogenase $(\alpha$-LAD), a component of 2-oxoacid dehydrogenase multienzyme complexes in the mitochondrial matrix, whereas approximately one-quarter is due to the electron transport dehydrogenase's flavin moiety (Kunz and Kunz 1985). The redox state of $\alpha$-LAD's flavin cofactor is in tight equilibrium with the mitochondrial $\mathrm{NAD}^{+} / \mathrm{NADH}$ pool. Therefore, the amount of flavoprotein autofluorescence is inversely related to the amount of mitochondrial metabolic activity. This is illustrated by the scheme (Kunz et al. 1997): 
Primary dehydrogenases $\rightarrow$ NADH (red., fluorescent) $/ \mathrm{NAD}^{+}$(oxid.) $\rightarrow$ Respiratory chain $\rightarrow \mathrm{O}_{2}$

$\uparrow \downarrow$

$$
\alpha-\mathrm{LAD}-\mathrm{H}_{2} \text { (red.) } / \alpha-\mathrm{LAD} \text { (oxid., fluorescent) }
$$

When metabolic activity is high, NADH predominates and non-fluorescent, reduced flavoprotein $\left(\alpha-\mathrm{LAD}-\mathrm{H}_{2}\right)$ is abundant. Similarly, uncouplers of oxidative phosphorylation, such as 2,4-dinitrophenol (DNP) and carbonyl cyanide $p$-(trifluoromethoxy)phenylhydrazone (FCCP), accelerate electron transport, thereby promoting flavoprotein oxidation and increased fluorescence. When metabolic activity is low (e.g., apoptosis), $\mathrm{NAD}^{+}$predominates and oxidized, fluorescent flavoprotein $(\alpha$-LAD) predominates. In the presence of the membrane-permeable mitochondrial substrate octanoate and the cytochrome oxidase (complex IV) inhibitor cyanide (which "backs-up" electrons in the respiratory chain), the respiratory chain linked flavoproteins are predominantly reduced, decreasing flavoprotein fluorescence. Thus, the inversely related autofluorescence intensities of $\mathrm{NAD}(\mathrm{P}) \mathrm{H}$ and flavoproteins are metabolic barometers in living cells.

In addition to mitochondrial flavoproteins, several cell types such as monocytes and neutrophils also possess flavoproteins in association with the NADPH oxidase complex, which produces superoxide anions that participate in host defense mechanisms (Babior 1999). The NADPH oxidase is made up of multiple components, including p47-phox, p67-phox, Rac, and p91phox, which is a flavoprotein. gp91 has binding sites for NADPH, FAD, and heme. Although the properties of purified NADPH oxidase components have been thoroughly studied in vitro, the operational nature and regulation of the oxidase in living cells is not well understood. Recently, we have reported various aspects of the substrate-level regulation of the NADPH oxidase. For example, the activity of the oxidase may be regulated by the availability of its substrate NADPH (Kindzelskii and Petty 2002; Kindzelskii et al. 1997, 2002; Petty 2000, 2001). Indeed, the oxidase is part of a larger dynamic regulatory system within neutrophils which leads to changes in the amplitude of NADPH oscillations and the amplitude of ROM production (Olsen et al. 2003). In addition, the blunted activation of neutrophils from pregnant women may be traced to retrograde translocation of glucose-6-phosphate dehydrogenase, which is part of the NADPH-producing hexose monophosphate shunt (Kindzelskii et al. 2002). Furthermore, using high-speed microscopy we have recently shown that $\mathrm{NAD}(\mathrm{P}) \mathrm{H}$ waves travel along the long axis of neutrophils and then release superoxide anions as a pulse at the leading edge (Kindzelskii and Petty 2002). In the present study we explore the redox state of flavoproteins in neutrophils and monocytes. In particular, we show that the flavoprotein component of the NADPH oxidase undergoes periodic reduction lasting $\sim 54 \mathrm{~ms}$; this work provides compelling evidence that the oxidase is only periodically transporting electrons within living cells. The implications of this behavior in host defense are considered.

\section{Materials and methods}

\section{Reagents}

FMLP ( $N$-formyl-met-leu-phe), diphenylene iodonium (DPI), rotenone, antimycin A, cyanide, azide, DNP, and FCCP were obtained from Sigma (St. Louis, Mo.).

\section{Cell preparation}

Neutrophils and monocytes were isolated from peripheral blood using Ficoll-Hypaque (Sigma) density gradient centrifugation at $300 \times g$ for $30 \mathrm{~min}$ at room temperature (Kindzelskii et al. 1997). The viability of the isolated neutrophils was always $>95 \%$, as assessed by trypan blue exclusion. Cells were suspended in HBSS (Hanks balanced salt solution) or PBS (phosphate-buffered saline) (Life Technologies, Grand Island, NY).

Immunofluorescence staining

After washing with PBS, adherent cells were fixed with cold $3.7 \%$ paraformaldehyde for $30 \mathrm{~min}$. Cells were rinsed once, then fresh $3.7 \%$ paraformaldehyde wash added for an additional hour. After washing with PBS, cells were permeabilized with $1 \%$ Brij-58 for $30 \mathrm{~min}$ at room temperature, followed by washing and fixation with $3.7 \%$ paraformaldehyde for $30 \mathrm{~min}$. Samples were labeled with a rabbit anti-gp91-phox antibody (Upstate Biotechnology, Lake Placid, NY) for $20 \mathrm{~min}$ at room temperature. Cells were washed then labeled with a rhodamine-conjugated mouse antirabbit $\mathrm{IgG}$ for $30 \mathrm{~min}$ at room temperature followed by extensive washing with PBS.

\section{Fluorescence imaging}

A fluorescence microscope with a quartz condenser, quartz objectives, and a mercury illumination source (Carl Zeiss, New York, NY) were employed. The microscope's bottom port was employed for fluorescence detection to minimize the number of optical components. This port was fiber-optically coupled to a cooled intensifier $\left(\sim-20^{\circ} \mathrm{C}\right)$, which was attached to a Peltier-cooled I-MAX-512 camera $\left(\sim-20^{\circ} \mathrm{C}\right)$ (Princeton Instruments, Princeton, NJ). Image acquisition was controlled by a high-speed Princeton ST-133 interface and a Stanford Research Systems (Sunnyvale, Calif.) DG-535 delay gate generator. For flavoprotein fluorescence imaging, a filter set comprising a $455 \mathrm{~nm}$ DF70 excitation filter, a $520 \mathrm{~nm}$ DF40 emission filter, and a $495 \mathrm{~nm}$ long-pass dichroic reflector was used. For rhodamine fluorescence imaging, a $540 \mathrm{~nm}$ DF20 excitation filter, a $590 \mathrm{~nm}$ DF30 emission filter, and a $560 \mathrm{~nm}$ long-pass dichroic reflector were used.

\section{Emission spectroscopy}

The system described in the previous paragraph was used to perform emission spectroscopy. However, in this case the bottom port of the microscope was fiber-optically coupled to the input of an 
Acton-150 (Acton Instruments, Acton, Mass.) imaging spectrophotometer (Petty et al. 2000). A ruled grating of 300 grooves $/ \mathrm{mm}$ was employed. A $455 \mathrm{~nm}$ DF70 excitation filter and a $495 \mathrm{~nm}$ longpass dichroic mirror were employed for illumination. The exit side was coupled to an I-MAX-512 camera. Winspec software was used for data management. The instrument was calibrated using known mercury lines.

\section{Excitation spectroscopy}

Excitation spectroscopy was performed using a Peltier-cooled PMT D104 system (Photon Technology, Lawrenceville, NJ) attached to Zeiss axiovert 35 (Zeiss) fluorescence microscope. A monochromator and a fiber-optically coupled xenon lamp were controlled by FeliX software (Photon Tech.). For excitation spectroscopy, a $495 \mathrm{~nm}$ long-pass dichroic mirror and a $520 \mathrm{~nm}$ DF40 emission filter were used. Excitation spectra were processed by FeliX software. Multiple excitation spectra were averaged with each accumulated using a $0.2 \mathrm{~s}$ integration time and a $1 \mathrm{~nm}$ step size.

\section{Results}

The present study examines the ability of flavoprotein autofluorescence to serve as a functional marker for leukocytes. In particular, we examine the hypotheses that the metabolism of leukocytes oscillate in time (mitochondrial flavoprotein emission) and that the NADPH oxidase (peripheral and granular flavoprotein emission) undergoes brief periods of reduction (or electron transport) in cells. We use two leukocyte model systems in these studies: monocytes and neutrophils. We use monocytes to study cell metabolism, whereas neutrophils, which rely primarily on glycolysis for energy production (Roos and Balm 1981), are used to examine the redox properties of the NADPH oxidase.

\section{Steady-state spectroscopy}

In the first series of experiments we determined the autofluorescence emission spectra of FAD, the flavin component of $\alpha$-LAD, and unlabeled human leukocytes using a $455 \mathrm{~nm}$ DF70 excitation filter in conjunction with a microscope and an imaging spectrophotometer apparatus (Petty et al. 2000). The autofluorescence emission spectra from 500 to $750 \mathrm{~nm}$ of unlabeled human monocytes and purified commercially available FAD are shown in Fig. 1A and Fig. 1B, respectively. Emission spectra exhibiting maxima at $530 \mathrm{~nm}$ are observed for both samples; extensive studies have shown that these emission features are observed for flavoproteins (e.g., Kunz 1986; Kunz and Kunz 1985; Kunz et al. 2002; Kuznetsov et al. 1998). To examine the excitation properties associated with the $530 \mathrm{~nm}$ emission, excitation spectroscopy was performed using a microfluorometry apparatus set to an emission wavelength of $530 \mathrm{~nm}$ with a $10 \mathrm{~nm}$ slit width. Figure 1C and Fig. 1D show the excitation spectra of monocytes and FAD, respectively. The excitation maximum of monocytes is $460 \mathrm{~nm}$ whereas that of FAD is $470 \mathrm{~nm}$. The slight red

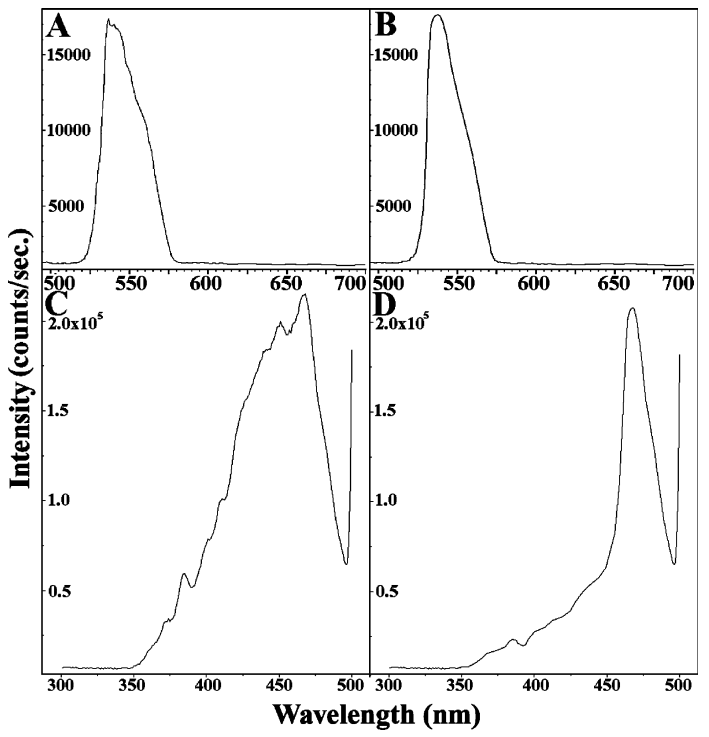

Fig. 1A-D Excitation and emission spectra of isolated cells and FAD. In panels $\mathbf{A}$ and $\mathbf{B}$ a microscope equipped with an imaging spectrophotometer was employed with a $\mathrm{Hg}$ lamp and a $455 \mathrm{~nm}$ DF70 filter for excitation. The fluorescence intensity is given at the ordinate and the wavelength is listed at the abscissa. Experiments were performed with an excitation microspectrophotometer apparatus using a $520 \mathrm{~nm}$ DF40 emission filter in panels $\mathbf{C}$ and $\mathbf{D}$ Panels $\mathbf{A}$ and $\mathbf{C}$ show spectra of cells while those of $\mathbf{B}$ and $\mathbf{D}$ show FAD $(n=3)$. The excitation spectrum of monocytes $(\mathbf{C})(n=8)$ is somewhat broader than that of purified FAD (D). However, the peak excitation wavelengths are similar. These findings are consistent with previous studies

shift and narrowing of the purified FAD spectrum is likely due to the fact that it is in a more hydrophilic environment than that of the FAD in association with the proteins and membranes found within living cells. Thus, the cell autofluorescence at $530 \mathrm{~nm}$ likely originates from flavoproteins.

It is well known that only oxidized flavoproteins are autofluorescent (e.g., Kunz 1986; Kunz and Kunz 1985; Kunz et al. 2002; Kuznetsov et al. 1998). Therefore we have performed a series of experiments using metabolic inhibitors to vary the redox state of mitochondrial flavoproteins, particularly $\alpha$-LAD and the electron transport dehydrogenase (Kunz and Kunz 1985). Figure 2 shows a series of control and experimental emission spectra collected in the absence and presence of various metabolic inhibitors. Figure $2 \mathrm{~A}$ shows the emission spectrum of an untreated cell. In the presence of $10 \mu \mathrm{M}$ rotenone, an inhibitor of the NADH dehydrogenase (complex I of the respiratory chain), and the mitochondrial substrate octanoate $(1 \mathrm{mM})$ for $5 \mathrm{~min}$ at $37^{\circ} \mathrm{C}$, the flavoprotein autofluorescence is significantly reduced, as indicated by the reduced peak height at $530 \mathrm{~nm}$ (Fig. 2B). However, octanoate alone had no effect on cellular autofluorescence (data not shown). This was expected because rotenone in the presence of octanoate is known to reduce flavoproteins (Kunz 1986). However, rotenone in the absence of octanoate increases flavoprotein emission of muscle tissue (Kuznetsov et al. 


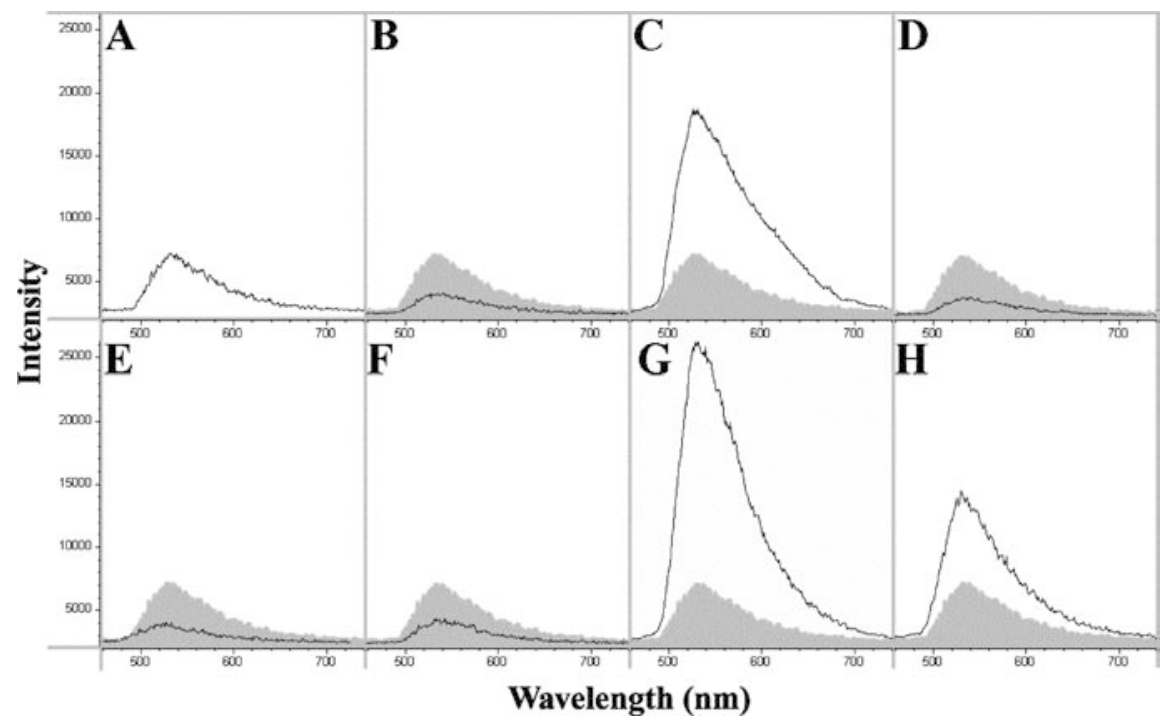

Fig. 2A-H Effect of metabolic inhibitors on emission spectra of human monocytes. Experiments utilized an imaging spectrophotometer attached to a microscope. The fluorescence intensity (ordinate) and wavelength (abscissa) are shown. A, untreated monocytes $(n>3)$. This spectrum is repeated as the lightly shaded area in panels $\mathbf{B}-\mathbf{H}$. B, $10 \mathrm{mM}$ rotenone; $\mathbf{C}, 10 \mathrm{mM}$ rotenone $+1 \mathrm{mM}$ octanoate; $\mathbf{D}, 1 \mathrm{mg} / \mathrm{mL}$ antimycin $\mathrm{A} ; \mathbf{E}, 5 \mathrm{mM}$ cyanide; F, $5 \mathrm{mM}$ azide; $\mathbf{G}, 1 \mathrm{mM}$ DNP; and $\mathbf{H}, 10 \mathrm{mM}$ FCCP. Conditions promoting flavoprotein oxidation increase flavoprotein emission, whereas conditions that lead to flavoprotein reduction decrease fluorescence

1998). This rather unusual feature was also confirmed in human monocytes. As shown in Fig. 2C, the autofluorescence of monocytes increases in the presence of only rotenone $(10 \mu \mathrm{M})$. To further confirm the role of cell metabolism in the regulation of the $530 \mathrm{~nm}$ autofluorescence, cells were incubated with octanoate and antimycin A $(1 \mu \mathrm{g} / \mathrm{mL})$, an inhibitor of the cytochrome $b / c 1$ complex (complex III). Similarly, the autofluorescence of cells was reduced (Fig. 2D). Additional experiments were performed in the presence of azide $(5 \mathrm{mM})$ or cyanide $(5 \mathrm{mM})$, two reagents known to act on the cytochrome $c$ oxidase (complex IV). Again, these reagents caused electrons to accumulate in the electron transport chain, thereby reducing more flavoprotein molecules and decreasing autofluorescence (Fig. 2E, F). Thus, autofluorescence is sensitive to conditions known to reduce mitochondrial flavoproteins.

Previous studies have demonstrated that uncouplers of oxidative phosphorylation, which dysregulate mitochondrial function and draw electrons through the respiratory chain, increase flavoprotein autofluorescence of cells (Kunz et al. 1997). We therefore incubated monocytes with DNP $(10 \mu \mathrm{M})$ and FCCP $(10 \mu \mathrm{M})$, two well-known uncouplers of oxidative phoshorylation. In both cases the autofluorescence intensity was significantly enhanced (Fig. 2G, H). Thus, conditions known to promote flavoprotein oxidation increase the autofluorescence intensity. These studies provide strong physical (spectroscopic) and functional (metabolic) evidence that much of the autofluorescence of cells at $530 \mathrm{~nm}$ originates from flavoproteins. These findings are consistent with prior studies of various cell types, including leukocytes (Heintzelman et al. 2000; Kunz 1986; Kunz and Kunz 1985; Kunz et al. 1993, 1997, 2002; Kuznetsov et al. 1998; Mayeno et al. 1992). However, one notable feature of these experiments (Fig. 2A-H) is that the flavoprotein emission could not be eliminated, which is consistent with the presence of a non-mitochondrial flavoprotein, such as the NADPH oxidase.

\section{Oscillations in flavoprotein intensity}

Previous studies have demonstrated that morphologically polarized neutrophils exhibit oscillations in membrane potential, calcium signals, metabolism, actin assembly, ROM production, etc. (for a review see Petty 2001). To examine the relationship between previously reported metabolic oscillations and flavoprotein autofluorescence, we studied $\mathrm{NAD}(\mathrm{P}) \mathrm{H}$ oscillations and flavoprotein autofluorescence of monocytes and neutrophils. Kinetic studies of flavoprotein intensity were performed using a microfluorometry apparatus. Untreated monocytes exhibited flavoprotein oscillations of $\sim 20 \mathrm{~s}$ (Fig. 3a), which match those of $\mathrm{NAD}(\mathrm{P}) \mathrm{H}$ (Fig. 3d). Monocytes activated with FMLP demonstrate $\sim 10 \mathrm{~s}$ oscillations (Fig. 3b), whereas those activated with interferon- $\gamma$ display high amplitudes and $\sim 20 \mathrm{~s}$ periods (Fig. 3c). The amplitudes and periods of these flavoprotein oscillations parallel those of $\mathrm{NAD}(\mathrm{P}) \mathrm{H}$ during the same experimental conditions (Fig. 3e, f) (Petty 2001). A longer time period flavoprotein oscillation ( $\sim 3$ min period) underlying the more rapid oscillations is more apparent in monocytes (Fig. 3a-c) than neutrophils (Fig. $4 \mathrm{a}-\mathrm{c}$ ). Thus, we now report that an additional marker of cell metabolism, the redox state of flavoproteins, oscillates in human cells.

We next examined the temporal relationship between these two oscillators. Figure $3 \mathrm{~g}$ shows a representative experiment where we switched between the optical 


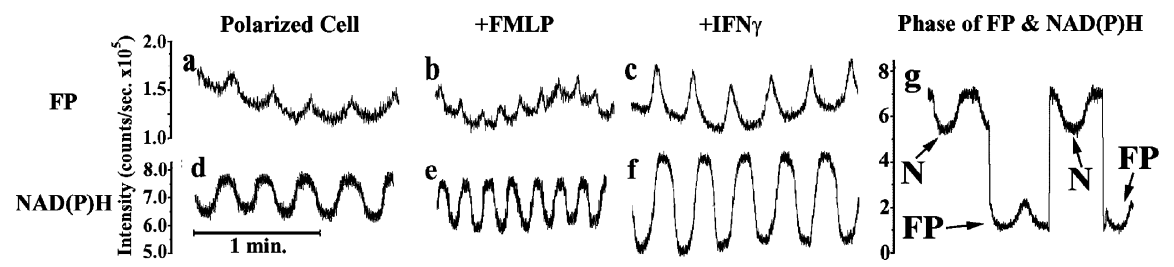

Fig. 3a-g Flavoprotein and NAD(P)H autofluorescence oscillations of monocytes. A microfluorometer was used to follow cellular autofluorescence over several min. Cells were monitored with the flavoprotein $(\mathbf{a}-\mathbf{c})$ or $\mathrm{NAD}(\mathrm{P}) \mathrm{H}(\mathbf{d}-\mathbf{f})$ autofluroescence set-ups. The oscillation periods of flavoproteins and $\mathrm{NAD}(\mathrm{P}) \mathrm{H}$ are similar for untreated polarized cells (a, d), FMLP-treated cells (b, e), and interferon- $\gamma$-treated cells (c, f). Interferon- $\gamma$ treatment also illustrates the fact that the amplitudes increase in parallel. In panel $\mathbf{g}$ the optical set-ups for imaging flavoprotein (labeled FP) and $\mathrm{NAD}(\mathrm{P}) \mathrm{H}$ (labeled $N$ ) emission were rapidly switched. These data indicate that the trough of the flavoprotein oscillation occurs during the peak of the $\mathrm{NAD}(\mathrm{P}) \mathrm{H}$ oscillation. For $\mathbf{a}-\mathbf{f}, n=4$; for $\mathbf{g}$, $n=3$

set-ups for detecting flavoprotein and NAD(P)H autofluorescence emission. The $\mathrm{NAD}(\mathrm{P}) \mathrm{H}$ and flavoprotein traces are indicated in this panel. The peaks in flavoprotein intensity occur at troughs of $\mathrm{NAD}(\mathrm{P}) \mathrm{H}$ intensity, which indicate that these two oscillators have phases differing by $180^{\circ}$. Given the manner in which flavoproteins respond to $\mathrm{NAD}(\mathrm{P}) \mathrm{H}$ levels (Eq. 1), this result is consistent with the literature. A similar series of observations were noted for neutrophils (Fig. 4), suggesting that these features are found in multiple types of leukocytes.

The flavoprotein oscillations described above are linked with cell metabolism. Addition of inhibitors such as cyanide and DNP, which have opposite effects on the redox state of mitochondrial flavoproteins, cause the amplitude of the oscillations to progressively disappear. This is illustrated by the kinetic studies shown in Fig. 5. These inhibitors change the redox state of cellular flavoproteins and within a few hundred seconds of addition to samples cause the oscillations to disappear, presumably by altering the feedback pathways participating in oscillations. Thus, metabolic inhibitors can

Fig. 4a-g Flavoprotein and NAD(P)H autofluorescence oscillations of neutrophils. A microfluorometer was used to monitor the flavoprotein $(\mathbf{a}-\mathbf{c})$ or $\mathrm{NAD}(\mathrm{P}) \mathrm{H}(\mathbf{d}-\mathbf{f})$ autofluroescence of neutrophils. The oscillatory properties of flavoproteins and $\mathrm{NAD}(\mathrm{P}) \mathrm{H}$ are similar for untreated polarized cells $(\mathbf{a}, \mathbf{d})$, FMLP-treated cells $(\mathbf{b}, \mathbf{e})$, and interferon- $\gamma$-treated cells $(\mathbf{c}, \mathbf{f})$. Interferon- $\gamma$ treatment also illustrates the fact that the amplitudes increase in parallel. In panel $\mathbf{g}$ the optical set-ups for imaging flavoprotein (labeled $F P$ ) and $\mathrm{NAD}(\mathrm{P}) \mathrm{H}$ (labeled $N$ ) emission were rapidly switched. These data indicate that the trough of the flavoprotein oscillation occurs during the peak of the NAD(P)H oscillation. $n=3$ affect both the average autofluorescent intensity and the oscillations in fluorescence intensity.

\section{Flavoprotein imaging}

We next sought to better define the intracellular locations of the flavoproteins. To address this issue, we performed a series of imaging experiments using the flavoprotein excitation and emission properties defined above. The relatively wide excitation filter was used because of the broad excitation spectrum of flavoproteins and to allow excitation by the bright mercury line at $436 \mathrm{~nm}$. Figure 6 shows autofluorescence micrographs of human monocytes. Untreated monocytes display a dim essentially uniform fluorescence throughout the cell and a punctate autofluorescence primarily found near the cell periphery (Fig. 6A, B), thus suggesting at least two flavoprotein compartments within these cells. To more fully characterize these compartments, monocytes were treated with metabolic inhibitors.

Figure 6C shows a fluorescence micrograph of monocytes treated with $10 \mathrm{mM}$ rotenone and $1 \mathrm{mM}$ octanoate. As expected, the cells become brighter (see Fig. $2 \mathrm{C}$ for quantitative data). Although the fluorescence increases throughout the cell, the punctate fluorescence does not appear to be affected significantly. On the other hand, addition of cyanide $(5 \mathrm{mM})$ reduced cellular autofluroescence, except for the punctate component (Fig. 6F). This suggests that the punctate or granular fluorescence is not associated with the electron transport chain of mitochondria. As a negative control, DPI, an inhibitor of the NADPH oxidase, was employed; as expected, this had no effect on cellular autofluorescence (Fig. 6I). We interpret the findings of Figs. 1, 2, 3 to indicate that much of the monocyte's flavoprotein autofluorescence arises from the mitochondrial electron transport chain, which can be found throughout the cytoplasm. In addition, the granular/ peripheral flavoprotein autofluorescence likely arises from the flavoprotein associated with the NADPH oxidase, which is located within granular and plasma membrane fractions.

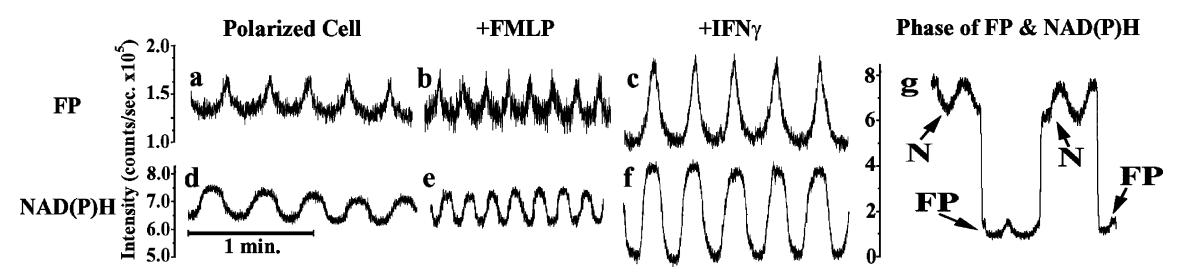




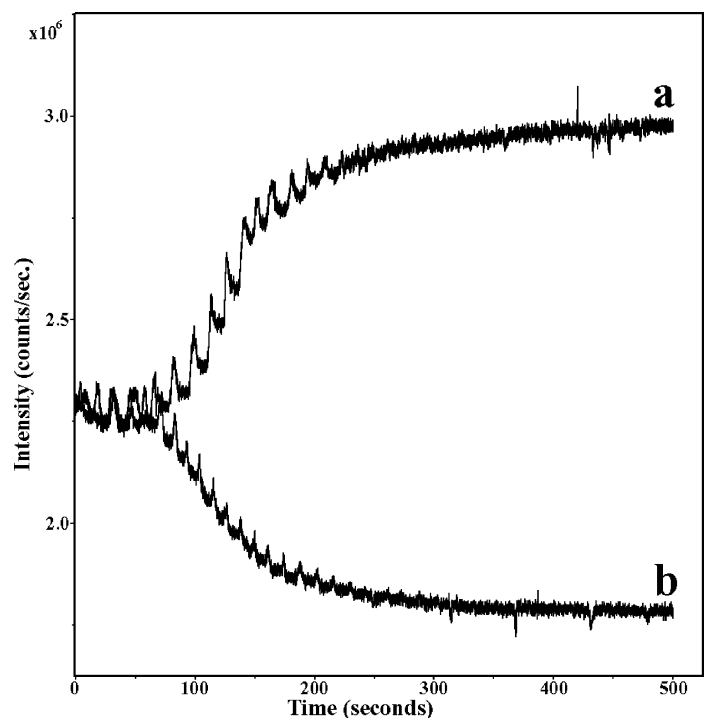

Fig. 5 Metabolic inhibitors also affect flavoprotein autoflurescence oscillations. Flavoprotein oscillations were detected as described in Fig. 3. However, in these experiments the metabolic inihibitors DNP (trace $a$ ) and cyanide (trace $b$ ) were added during kinetic observations at $37^{\circ} \mathrm{C}$. As the data indicate, these metabolic inhibitors can promote the oxidation or reduction of the flavoprotein, but the oscillatory intensity decays within several hundred seconds, thus confirming the link between the oscillations, per se, and cell metabolism

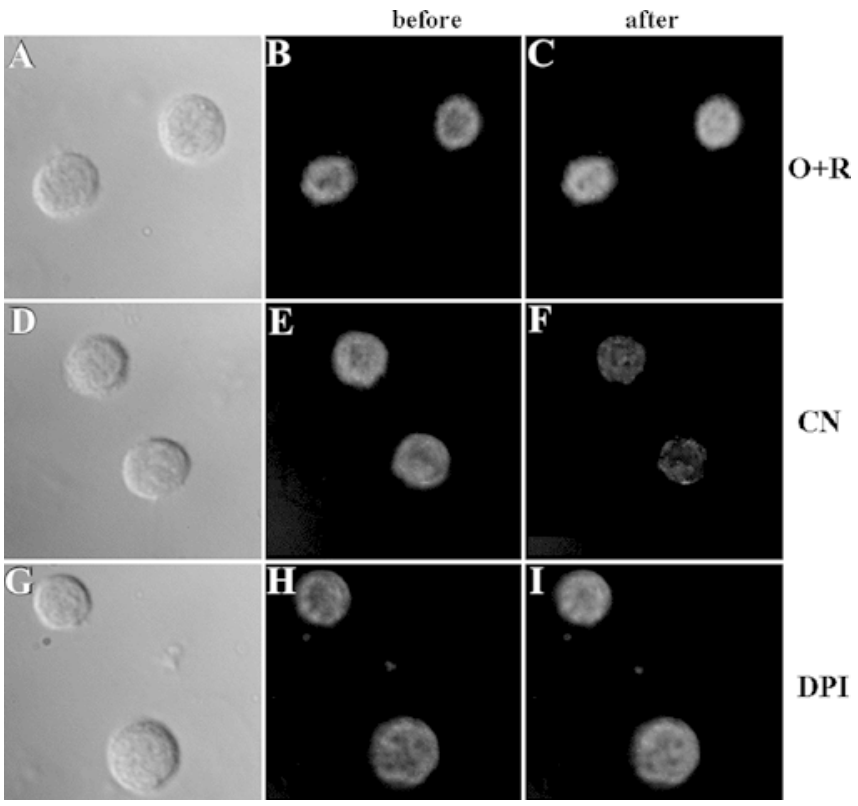

Fig. 6A-I Microscopic imaging of monocyte flavoprotein autofluorescence. Flavoprotein autofluorescence of monocytes was imaged during a variety of conditions. DIC photomicrographs $(\mathbf{A}, \mathbf{D}, \mathbf{G})$ and fluorescence photomicrographs $(\mathbf{B}, \mathbf{C}, \mathbf{E}, \mathbf{F}, \mathbf{H}, \mathbf{I})$ are shown. Panels $\mathbf{B}, \mathbf{E}$, and $\mathbf{H}$ show cells before treatment with inhibitors, whereas $\mathbf{C}, \mathbf{F}$, and $\mathbf{I}$ show cells after exposure to $10 \mathrm{mM}$ rotenone $+1 \mathrm{mM}$ octanoate $(\mathbf{C}), 5 \mathrm{mM}$ potassium cyanide $(\mathbf{F})$, and DPI (I). Although cyanide dramatically decreases total cellular autofluorescence levels, it does not have effect the peripheral granular fluorescence of cells. Magnification $\times 1120 ; n=3$
Immunofluorescence imaging of the NADPH oxidase

To provide further data supporting the NADPH oxidase as a source of flavoprotein autofluorescence in neutrophils, cells were stained with an antibody specific for the NADPH oxidase. Neutrophils were first photographed using the excitation and emission set-up described in the preceding paragraph. Cells were fixed, stained with the anti-gp-91-phox, a major component of the NADPH oxidase, then labeled with a rhodamineconjugated second-step antibody. When flavoprotein autofluorescence was imaged, some diffuse cytoplasmic staining was found in addition to strong peripheral staining of granules and membranes (Fig. 7B). Much of the peripheral flavoprotein emission corresponds to NADPH oxidase, as judged by the anti-gp91-phox staining pattern (Fig. 7C). This is further supported by resonance energy transfer experiments, which were performed as previously described (Kindzelskii et al. 1997). Resonance energy transfer between the flavoprotein and the rhodamine labeled gp91-phox can be observed (Fig. 7D), which suggests that the FAD moiety and the second-step antibody molecule are within $7 \mathrm{~nm}$ of one another. Thus, by selecting peripheral granules and membrane for analysis, it should be possible to observe changes in the redox state of the NADPH oxidase since a significant fraction of the flavoprotein emission is due to the oxidase.

\section{Time-resolved spectroscopy}

Having thoroughly examined the origins of flavoprotein fluorescence in leukocytes, we next examined the spectroscopic properties of the flavoprotein emission associated with the plasma membrane and granular fractions. In particular, we sought to test the hypothesis that the NADPH oxidase is active for only brief periods of time, as suggested by previous experiments from this laboratory (Kindzelskii and Petty 2002). To test this concept, we employed time-resolved spectroscopy of flavoprotein autofluorescence. These experiments employed peripheral blood neutrophils to minimize the contribution of mitochondrial flavoproteins. In addition, we examined flavoprotein emission in the region of the neutrophil's lamellipodium, since this further reduces mitochondrial contributions and this cellular region has been previously associated with the release of superoxide anions (Kindzelskii and Petty 2002), the chemical product of the NADPH oxidase. Figure 8 shows representative time-resolved spectroscopy experiments of neutrophil lamellipodia. Although the cells were morphologically polarized, they were not activated by immunological stimulants, which may change the properties of the oxidase. The spectroscopy experiments of Fig. 8A show that NADPH oxidase-related flavoprotein undergoes brief periods of reduction; in this case flavoprotein reduction, or electron trafficking across the membrane, lasted about $54 \mathrm{~ms}$ (a value of $54 \pm 2 \mathrm{~ms}$ was 


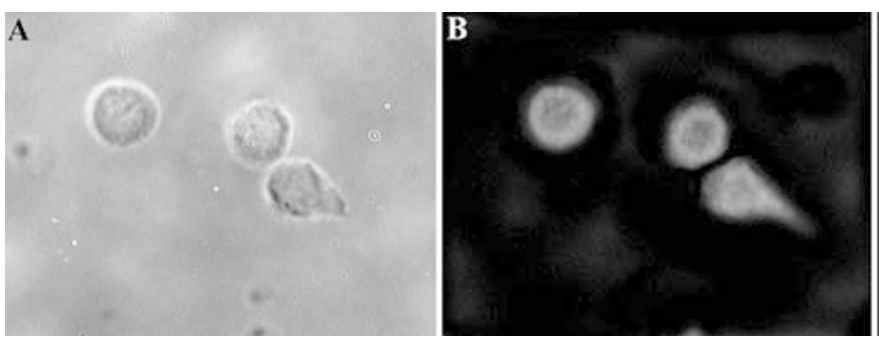

Fig. 7A-D Co-localization of flavoprotein autofluorescence and the gp-91-phox component of the NADPH oxidase. DIC (A) and fluorescence (B, C) micrographs of neutrophils are shown. Flavoprotein autofluorescence is shown in panel $\mathbf{B}$. Panel $\mathbf{C}$ shows the same cells stained with anti-gp-91-phox antibody. The flavoprotein autofluorescence and the NADPH oxidase staining patterns overlap. The relationship between the peripheral flavoprotein and the NADPH oxidase was further confirmed by resonance energy transfer experiments. The flavoprotein moiety was excited and rhodamine fluorescence of the associated with NADPH oxidase was monitored (panel D). The presence of energy transfer indicates that the flavin moiety is within $7 \mathrm{~nm}$ of the antigp-91-phox antibody, as expected. Magnification $\times 910$

obtained during four separate experiments). Although decreased flavoprotein emission could be observed in the presence of cyanide, the NADPH oxidase inhibitor DPI (Cross and Jones 1986) blocked the periodic reduction of this flavoprotein component (Fig. 8B). These high-speed

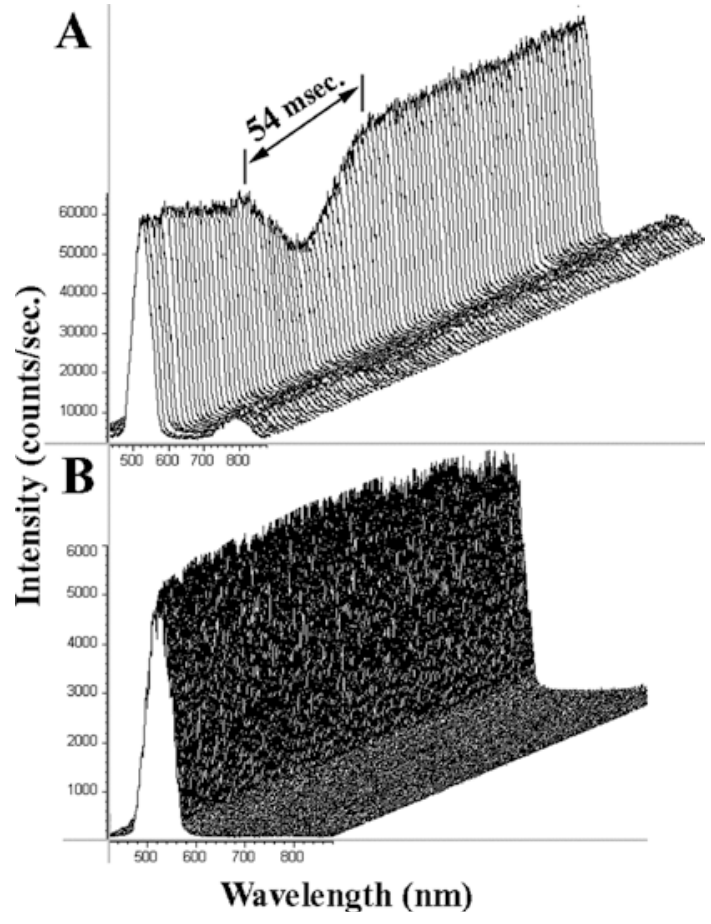

Fig. 8A, B Time-resolved emission spectrophotometry of peripheral neutrophil flavoprotein autofluorescence. Data were acquired from the region of the lamellipodium of the cell. Emission spectra were acquired for $1 \mathrm{~ms}$, then downloaded from the CCD chip, which required $1 \mu \mathrm{s}$; this cycle was repeated to obtain the series of spectra shown. Panel A shows an untreated cell; note the brief decrease in fluorescence intensity of the flavoprotein. However, if cells are treated with the NADPH oxidase inhibitor DPI at $1 \mathrm{mM}$, no reduction in flavoprotein intensity is observed (panel B). $n=4$
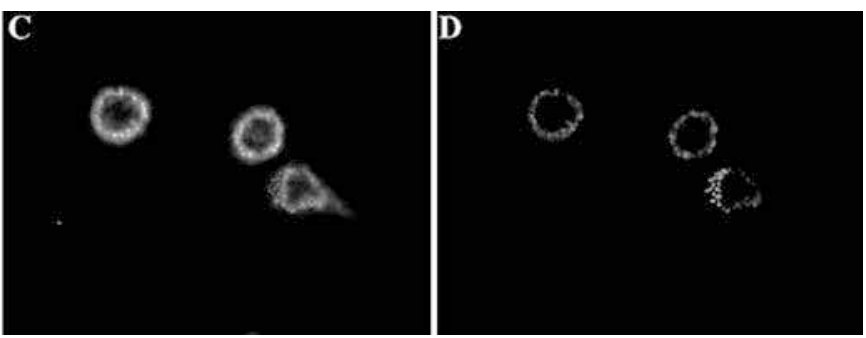

spectroscopy experiments confirm that a significant fraction of the peripheral flavoprotein fluorescence is associated with the NADPH oxidase and demonstrate that electrons reduce the flavoprotein for brief periods of time.

\section{Discussion}

Previous studies have examined flavoprotein autofluorescence of eosinophils, neutrophils, and mononuclear leukocytes using HPLC and emission spectroscopy (Heintzelman et al. 2000; Mayeno et al. 1992). The present study employs efficient microscopic systems and detectors to collect emission and excitation spectra and images from individual living cells. Our studies confirmed that much of the cellular autofluorescence at $530 \mathrm{~nm}$ is associated with flavoproteins of cellular metabolism, as judged by the metabolic inhibitors rotenone, antimycin A, azide, cyanide, DNP, and FCCP. However, the autofluorescence was not uniquely associated with cell metabolism since the flavoprotein signal could not be eliminated using inhibitors of electron transport. Fluorescence microscopy revealed that autofluorescence of the plasma membrane and a largely peripheral punctate fluorescence, primarily granular in nature, are not affected by metabolic inhibitors. Inasmuch as monocytes contain flavoproteins associated with mitochondria and the NADPH oxidase, we suggest that the respiratory electron transport chain-independent autofluorescence of the plasma membrane and granules is due to the NADPH oxidase. This is consistent with the fact that the NADPH oxidase is found in the plasma membrane and intracellular granules.

The autofluorescence of flavoproteins and $\mathrm{NAD}(\mathrm{P}) \mathrm{H}$ have been employed in the in vitro microscopic evaluation of cell metabolism. Kunz and colleagues (Kunz 1986; Kunz and Kunz 1985; Kunz et al. 1993, 1997, 2002; Kuznetsov et al. 1998) have extensively characterized the mitochondrial flavoprotein responses in a variety of conditions, including clinical disorders. This laboratory (Kindzelskii and Petty 2002; Petty and Kindzelskii 2001; Petty et al. 2000) has used both microfluorometry and high-speed imaging to evaluate intracellular NAD $(\mathrm{P}) \mathrm{H}$ metabolism. These studies have revealed NAD $(\mathrm{P}) \mathrm{H}$ oscillations and traveling $\mathrm{NAD}(\mathrm{P}) \mathrm{H}$ waves within living cells. These propagating $\mathrm{NAD}(\mathrm{P}) \mathrm{H}$ waves were anticipated by the work of Turing, Prigogine, Hess, and others, then confirmed by our studies using high-speed imaging (Petty et al. 2000). Thus, the 
fluorescence of flavoproteins and $\mathrm{NAD}(\mathrm{P}) \mathrm{H}$ have been shown to be useful in the in vitro analysis of cell metabolism and its self-organized behavior. Our previous studies have focused on the oxidase's substrate, NADPH, and the production of its product superoxide anions. We have now directly examined the metabolic kinetics of flavoprotein activity in migrating neutrophils and the fast reaction dynamics of the NADPH oxidase in living cells by monitoring the autofluorescence of its associated flavin.

Previous studies have shown that in addition to the relatively slow metabolic oscillations of $\sim 3 \mathrm{~min}$ expressed by many cells and tissues, additional higher frequency components can also be present (Goldbeter 1996; Hess and Boiteux 1971; Petty 2001; Vern et al. 1988). Morphologically polarized neutrophils can display $\mathrm{NAD}(\mathrm{P}) \mathrm{H}$ oscillation periods of about $10 \mathrm{~s}$ and $20 \mathrm{~s}$ in the activated and non-activated states, respectively. We now show that these higher frequency components are also expressed by mitochondrial flavoproteins, which might be expected based upon the relatively close coupling of $\mathrm{NADH}$ and flavoprotein redox states (Eq. 1). Importantly, changes in the frequency and amplitude of NAD(P)H oscillations are also reflected in the flavoprotein intensity oscillations, although the oscillations differ in both relative phase and waveform. The relative contributions of the $\alpha$-LAD and electron transport dehydrogenase to these metabolic oscillations are unknown.

We have recently shown that metabolism is not uniform within polarized neutrophils at short time-scales, but rather takes the form of propagating longitudinal metabolic waves (Kindzelskii and Petty 2002; Petty and Kindzelskii 2001; Petty et al. 2000). Thus, the concentration of $\mathrm{NAD}(\mathrm{P}) \mathrm{H}$ varies spatio-temporally, with the lamellipodium of the cell periodically exposed to low and then, for brief periods of time, high concentrations of NAD $(\mathrm{P}) \mathrm{H}$. Our recent study (Kindzelskii and Petty 2002) indicated that these traveling $\mathrm{NAD}(\mathrm{P}) \mathrm{H}$ waves are decoded by the NADPH oxidase at the front of polarized neutrophils to create a brief highly concentrated pulse of superoxide anions. One implication of this finding is that the NADPH oxidase is active for relatively short periods of time, which has heretofore not been examined. To directly test this concept, we have monitored the redox state of the NADPH oxidase by following kinetically the autofluorescence emission of its flavoprotein component using high-speed spectrophotometry. These studies have shown that the flavoprotein is reduced for approximately $54 \mathrm{~ms}$, which approximates the length of time the $\mathrm{NAD}(\mathrm{P}) \mathrm{H}$ wave lingers in the region of the lamellipodium. Importantly, an inhibitor of electron trafficking through the NADPH oxidase, DPI, also inhibits the reduction of the flavoprotein and its change in autofluorescence. Thus, the NADPH oxidase is only transferring electrons for brief time periods during superoxide production.

Is it reasonable that the NADPH oxidase is active for brief time intervals? This single-electron reduction reaction produces $6 \mathrm{nmol}$ superoxide/10 min per $10^{6}$ cells (Schrenzel et al. 1998), which gives a rate of $\sim 10^{8}$ electrons/min per cell. Since eight metabolic waves arrive at the lamelliodium each minute, we have $\sim 10^{7}$ electrons/ pulse. This assumes that all of the superoxide is produced during the pulse, which is an approximate but conservative assumption. Although there are many copies of the NADPH oxidase in neutrophils, as visualized in Fig. 5, it is difficult to know the fraction of the NADPH oxidase molecules that are producing superoxide anions in a given cell. We again conservatively estimate that only a small fraction, roughly $\sim 10^{4} \mathrm{NADPH}$ oxidase molecules, are active. This suggests that about $\sim 1000$ electrons traffic through the oxidase with each pulse (or $10^{4}$ averaged over a second). One implication of this is that an electron would spend only $0.05 \mathrm{~ms}$ in transit through the oxidase. Thus, the residence time of electrons in the oxidase is thousands of times longer than those of cofactors in the photosynthetic apparatus (Petty 1993). The mitochondrial NADH dehydrogenase has a specific activity of $1 \mu \mathrm{mol}$ NADH oxidized/min per $\mathrm{mg}$ for mitochondrial particles (Vinogradov 1998). Using $\sim 1 \%$ as the NADH dehydrogenase protein fraction present in these particles and $10^{6}$ as its molecular weight, and taking into consideration that two electrons traffic per $\mathrm{NADH}$, one can estimate that 3000 electrons/s can move through the NADH oxidase (or $\sim 0.3 \mathrm{~ms} /$ electron). This is consistent with other estimates of the turnover number of this enzyme (Gupte et al. 1984). Thus, the rate of electron flux through the NADPH oxidase is within an order of magnitude of that found for the NADH dehydrogenase. Therefore, our findings are consistent with the rates of electron trafficking through other known biological systems.

Our findings support the concept that dynamic intracellular reaction schemes are an intrinsic element of living cells. However, these dynamic events generally require spectroscopic tools to dissect their behavior. Not only will these biophysical studies lead to a more comprehensive understanding of the living chemistry of cells, but they may also lead to new classes of drugs aimed at influencing cellular chemical dynamics, not necessarily the binding of a drug to any particular protein or DNA sequence.

\section{References}

Babior BM (1999) NADPH oxidase: an update. Blood 93:1464 1476

Cross AR, Jones OT (1986) The effect of the inhibitor diphenylene iodonium on the superoxide-generating system of neutrophils. Specific labelling of a component polypeptide of the oxidase. Biochem J 237:111-116

Goldbeter A (1996) Biochemical oscillations and cellular rhythms. Cambridge University Press, Cambridge, UK

Gupte S, Wu ES, Hoechli L, Hoechli M, Jacobson K, Sowers AE, Hackenbrock CR (1984) Relationship between lateral diffusion, collision frequency, and electron transfer of mitochondrial inner membrane oxidation-reduction components. Proc Natl Acad Sci USA 81:2606-2610 
Heintzelman DL, Lotan R, Richards-Kortum RR (2000) Characterization of the autofluorescence of polymorphonuclear leukocytes, mononuclear leukocytes and cervical epithelial cancer cells for improved spectroscopic discrimination of inflammation from dysplasia. Photochem Photobiol 71:327-332

Hess B, Boiteux A (1971) Oscillatory phenomena in biochemistry. Annu Rev Biochem 40:237-258

Kindzelskii AL, Petty HR (2002) Apparent role of traveling metabolic waves in periodic oxidant release by living cells. Proc Natl Acad Sci USA 99:9207-9212

Kindzelskii AL, Eszes MM, Todd RF III, Petty HR (1997) Proximity oscillations of complement receptor type 4 and urokinase receptors on migrating neutrophils are linked with signal transduction/metabolic oscillations. Biophys J 73:1777-1784

Kindzelskii AL, Huang JB, Chaiworapongsa, T, Kim YM, Romero R, Petty HR (2002) Pregnancy alters glucose-6-phosphate dehydrogenase trafficking, cell metabolism and oxidant release of maternal neutrophils. J Clin Invest 110:1801-1811

Kunz D, Luley C, Winkler K, Lins H, Kunz WS (1997) Flow cytometric detection of mitochondrial dysfunction in subpopulations of human mononuclear cells. Anal Biochem 246:218-224

Kunz D, Winkler K, Elger CE, Kunz WS (2002) Functional imaging of mitochondrial redox state. Methods Enzymol 352:135-150

Kunz WS (1986) Spectral properties of fluorescent flavoproteins of isolated rat liver mitochondria. FEBS Lett 195:92-96

Kunz WS, Kunz W (1985) Contribution of different enzymes to flavoprotein fluorescence of isolated rat liver mitochondria. Biochim Biophys Acta 841:237-246

Kunz WS, Kuznetsov AV, Schulze W, Eichhorn K, Schild L, Striggow F, Bohnensack R, Neuhof S, Grasshoff H, Neumann HW, Gellerick FN (1993) Functional characterization of mitochondrial oxidative phosphorylation in saponin-skinned human muscle fibers. Biochim Biophys Acta 1144:46-53

Kuznetsov AV, Mayboroda O, Kunz D, Winkler K, Schubert W (1998) Functional imaging of mitochondria in saponin-permeabilized mice muscle fibers. J Cell Biol 140:1091-1099
Mayeno AN, Hamann KJ, Gleich GJ (1992) Granule-associated flavin adenine dinucleotide (FAD) is responsible for eosinophil autofluorescence. J Leukocyte Biol 51:172-175

Olsen LF, Kummer U, Kindzelskii AL, Petty HR (2003) A model of the oscillatory metabolism of activated neutrophils. Biophys J 84:69-81

Petty HR (1993) Molecular biology of membranes. Plenum Press, New York

Petty HR (2000) Oscillatory signals in migrating neutrophils: effects of time-varying chemical and electrical fields. In: Walleczek $\mathbf{J}$ (ed) Self-organized biological dynamics and nonlinear control by external stimuli. Cambridge University Press, Cambridge, UK, pp 173-192

Petty HR (2001) Neutrophil oscillations: temporal and spatiotemporal aspects of cell behavior. Immunol Res 23:125-134

Petty HR, Kindzelskii AL (2001) Dissipative metabolic patterns respond during neutrophil transmembrane signaling. Proc Natl Acad Sci USA 98:3145-3149

Petty HR, Worth RG, Kindzelskii AL (2000) Imaging sustained dissipative patterns in the metabolism of individual living cells. Phys Rev Lett 84:2754-2757

Roos D, Balm AJM (1980) The oxidative metabolism of monocytes. In: Sbarra AJ, Strauss RR (eds) The reticuloendothelial system: a comprehensive treatise. Plenum Press, New York, pp 189-229

Schrenzel J, Serrander L, Banfi B, Nusse O, Fouyouzi R, Lew DP, Demaurex N, Krause KH (1998) Electron currents generated by the human phagocyte NADPH oxidase. Nature 392:734-737

Vern BA, Schuette WH, Leheta B, Juel VC, Radulovachi M (1988) Low-frequency oscillations of cortical oxidative metabolism in walking and sleep. J Cerebral Blood Flow Metab 8:215-226

Vinogradov AD (1998) Catalytic properties of the mitochondrial NADH-ubiquinone oxidoreductase (complex I) and the pseudo-reversible active/inactive enzyme transition. Biochim Biophys Acta 1364:169-185 